\title{
Living Donor Transplantation of Kidneys with Fibromuscular Dysplasia: Indications, Surgical Techniques and Long Term Results in 11 Cases
}

\author{
Luisa Berardinelli*, Claudio Beretta and Antenore Giussani \\ Unit of General Surgery and Kidney Transplantation, Fondazione IRCCS Ca' Granda Ospedale Maggiore Policlinico - Milan, Italy
}

\section{Introduction}

Due to the limited availability of organs for transplantation, the criteria for the acceptance of donor kidneys have been extended to include donations from expanded criteria donors either Deceased Donors (DD) or Living Donors (LD).

The presence of Fibromuscular Dysplasia (FMD), an arterial disorder characterized by "string-of-beads" of the renal artery, was one of the abnormalities which had previously excluded the acceptance of the potential donors.

The incidence of FMD in potential LDs is observed in a range from $2 \%$ to $6.6 \%$ with a female predominance [1]; it is the second most common anatomical abnormality after multiple renal arteries. FMD still represents a challenge for any decision to harvest the kidney, particularly from a $\mathrm{LD}$, due to the potential risk to develop a renovascular hypertension and/or FMD either in the remnant kidney after donation [2,3,1,4] or in the recipient's graft [5-7]. Moreover, the problem may only arise at the operative stage since early forms of FMD give rise to no or mild radiographic evidence. This 'mild' radiographic evidence has been described by Indudhara [2] as irregularity of the arterial wall without significant stenosis.

The aim of this study was to review the recipient and donor outcomes in 11 kidney transplants using kidneys affected by FMD and surgical skills adopted to decrease post-operative risks of failure.

\section{Patients and Methods}

From May 22, 1969 up to November 30,2011, 2,989 kidneys had been transplanted, as first (2,760), second (219) or third (10) graft at Policlinico University Hospital in Milan. In the meantime the hospital in question had become Ca' Granda Foundation, IRCCS. 2,617 of these grafts came from deceased donors and 372 from living donors.

A consecutive series of the latest LD transplants, a total of 273 carried out between February 1983 and October 2011 and whose recipients were all treated with Calcineurin Inhibitors (CNI), was retrospectively examined using our Transplant Unit database in order to assess the incidence of FMD in the LD grafts, the criteria on which the harvesting decision was based, the corrective surgery adopted to facilitate and safeguard the transplant, the outcome in the recipient of the graft affected by this disease and lastly, the early/long term outcome in the living donors with regard to "de novo" onset of FMD in the remnant kidney and/or of renovascular hypertension.

The candidates for donation were all evaluated through the obtainment of a complete medical history followed by physical examination, laboratory tests, cardiac evaluation and other tests aimed at detecting potential risk factors.

Color flow duplex imaging and dynamic renal scintigraphy were routinely carried out to identify the smaller organ or one with lower GFR, which could be harvested.

Preoperative assessment of the renal anatomy was made using standard renal arteriography through a femoral approach in 7 LDs out of the 11 under discussion. Nowadays Computed Tomographic Angiography (CTA) with vascular reconstruction is adopted as a reliable, non-invasive tool for the detection of multiple arteries and vascular anomalies. In $3 \mathrm{LDs}$, both angiographic tests were performed at the request of the transplant surgeon. This was because CTA was still a new tool and a comparison was considered necessary.

11 patients, out of the $273 \mathrm{LD}$ transplants performed since 1983, received kidneys with FMD.

The size of the kidney, the renal function at dynamic renal scintigraphy, the presence of multiple vessels or a mildly diseased artery, early branching and variants of lower urinary tract were all matter for the decision to harvest the kidney and for the choice of the site. In case of bilateral normality, the right side is our preferential choice.

Complete informed consent regarding the risks and benefits by donor and recipient was obtained, evidencing the major risk which an abnormal vascular supply or elevated blood pressure can imply.

Live donor nephrectomy was performed through an open subcostal extraperitoneal mini-incision $(8-10 \mathrm{~cm})$, that we have adopted since 1996. This surgical approach offers the same advantages of a handassisted video laparoscopic (VLS) technique, without the disadvantages typical of the VLS- nephrectomy, namely dependence on long learning period, longer operating time, longer warm ischemia times, shorter length of vessels, problems for right side kidney, more restrictive criteria of selection, high instrument costs and the significantly higher rate of major complications for both the donor and the recipient.

The grafts, coming from the right side (8 cases) or from the left side (3 cases) of the LDs were implanted in the right or left iliac fossa of the recipients, depending on the clinical situation at the time (previous removal of a polycystic kidney, doppler ultrasound findings of the atherosclerotic iliac artery or stenosis of iliac vein, ...).

The venous anastomosis was made by first, suturing the renal vein end-to-side to the external recipient iliac vein. In one case, two renal veins of similar diameter had been harvested with a small patch of the donor vena cava and anastomosed to the external iliac vein.

The techniques employed for treating the FMD and the indications for the reconstruction at bench are described below. The ureter was

${ }^{*}$ Corresponding author: Luisa Berardinelli, Unit of General Surgery and Kidney Transplantation, Fondazione IRCCS Ca' Granda Ospedale Maggiore PoliclinicoMilan, Italy, E-mail: luisa.berardinelli@unimi.it

Received January 21, 2012; Accepted July 12, 2012; Published July 16, 2012

Citation: Berardinelli L, Beretta C, Giussani A (2012) Living Donor Transplantation of Kidneys with Fibromuscular Dysplasia: Indications, Surgical Techniques and Long Term Results in 11 Cases. J Nephrol Ther S4:008. doi:10.4172/21610959.S4-008

Copyright: (c) 2012 Berardinelli L, et al. This is an open-access article distributed under the terms of the Creative Commons Attribution License, which permits unrestricted use, distribution, and reproduction in any medium, provided the original author and source are credited. 
Citation: Berardinelli L, Beretta C, Giussani A (2012) Living Donor Transplantation of Kidneys with Fibromuscular Dysplasia: Indications, Surgical Techniques and Long Term Results in 11 Cases. J Nephrol Ther S4:008. doi:10.4172/2161-0959.S4-008

Page 2 of 6

reimplanted using in all cases an extravesical technique with antireflux tunnel.

Immunosuppression consisted of cyclosporine as main drug (3 patients), assigned as triple therapy with prednisone and azathioprine (2) or MMF (1). The other 8 recipients received tacrolimus with MMF. Induction therapy with basiliximab at day 0 and 4 was employed in the last 10 of these recipients.

Rejection crises were treated with methyl-prednisolone $(10 \mathrm{mg} / \mathrm{Kg}$ for three days). OKT3 antibodies were used between 1985 and 1999 for steroid resistant cases, subsequently replaced by thymoglobulin.

The post-operative outcome of both donors and recipients as well as any onset of hypertension or FMD are reported. The pre- and early/long term post-donation serum creatinine levels are reported in the LDs, as well as pre- and post-donation glomerular filtration ratio, which was calculated using the Modification of Diet in Renal Disease (MDRD) system [8].

We compared the results of graft survival rates with those of $117 \mathrm{LD}$ adult recipients matched for sex, age, original diseases, characteristics of the donors and transplant period who received a "healthy" graft.

\section{Results}

In the consecutive series of LD candidates examined between 1983 and 2011, 15 cases of FMD have been observed: we excluded from donation, 4 LDs showing frank signs of "moderate" FMD, even in asymptomatic candidates, because of known tendency to bilateral involvement and possible development of the disease in the remnant or in donated kidney. In 11 cases we used LD kidneys with mild [2] FMD, that had been evidenced at angiographic findings in only 3 cases, whilst in the other 8 patients it was an intra-operative surprise.

The donors, 9 females and 2 males, aged from 48 to 68 years (mean $56.1 \pm 7.5$ years) were apparently healthy.

Seven of them showed normal blood pressure (BP), whilst 4 $(36.3 \%)$ were affected by mild arterial hypertension (mean systolic blood pressure $141 \mathrm{mmHg}$, mean diastolic blood pressure $82 \mathrm{mmHg}$ ), but only one of them was taking a low dosage angiotensin II receptor antagonist. The mean pre-donation value of Serum Creatinine was 0.77 \pm 0.09 (range $0.6-1.0$ ) and the value of MDRD was $83.7 \pm 10.4$ (range 74-108).

The mean age of recipients was $37.3 \pm 12.8$ (range $19-65$ ). Other clinical characteristics of the LDs affected by FMD and the relative recipients can be observed in Table 1 and in Table 2 .

\begin{tabular}{|l|l|}
\hline Characteristic & Value \\
\hline Sex, female/ male $\mathrm{n}^{\circ},(\%)$ & $9 / 2(81.8 / 18.2)$ \\
\hline $\begin{array}{l}\text { Age, mean } \pm \mathrm{SD}, \mathrm{yrs} \\
\text { (range) }\end{array}$ & $\begin{array}{l}56.1 \pm 7.5 \\
(48-68)\end{array}$ \\
\hline $\begin{array}{l}\text { Pre-donation Serum Creatinine, } \mathrm{mg} / \mathrm{dL} \\
\text { mean } \pm \mathrm{SD} \\
\text { (range) }\end{array}$ & $0.77 \pm 0.09$ \\
$(0.6-1.0)$
\end{tabular}

Table 1: Characteristics of 11 Living Donors with unilateral fibromuscular dysplasia.

\begin{tabular}{|l|l|}
\hline Characteristic & Value \\
\hline Sex, female / male, $\mathrm{n}^{\circ}$ & $5 / 6$ \\
\hline $\begin{array}{l}\text { Age, mean } \pm \mathrm{SD} \text {, yrs } \\
\text { (range) }\end{array}$ & $37.3 \pm 12.8$ \\
\hline End stage renal disease: & $(19-65)$ \\
Polycystic & 4 \\
Glomerulonephritis & 2 \\
Systemic Lupus Erythematosus & 2 \\
Pyelonephritis & 1 \\
Schönlein-Henoch & 1 \\
Focal glomerulosclerosis & 1 \\
Pre-TX dialysis months, mean \pm SD & $28.1 \pm 14.2$ \\
(range) & $(0-56)$ \\
\hline
\end{tabular}

Table 2: Characteristics of 11 recipients of LD-graft with fibromuscular dysplasia

\begin{tabular}{|l|l|}
\hline Characteristic & Value \\
\hline Side of kidney with FMD, Right/Left, $\mathrm{n}^{\circ}$ & $8 / 3$ \\
\hline $\begin{array}{l}\text { Serum Creatininemia at discharge, } \mathrm{mg} / \mathrm{dL} \\
\text { mean } \pm \text { SD } \\
\text { (range) }\end{array}$ & $\begin{array}{l}1.2 \pm 0.1 \\
(1.1-1.3)\end{array}$ \\
\hline $\begin{array}{l}\text { MDRD at discharge, mean } \pm \mathrm{SD}: \\
\text { (range) }\end{array}$ & $\begin{array}{l}50.9 \pm 5.6 \\
(43-64)\end{array}$ \\
\hline $\begin{array}{l}\text { Hypertension } 4 \text { yrs after donation, } \% \\
\text { MDRD } 4 \text { yrs after donation, mean } \pm S D: \\
\text { (range) }\end{array}$ & $36.3 \%$ \\
\hline
\end{tabular}

Table 3: Results in 11 LDs with unilateral fibromuscular dysplasia.

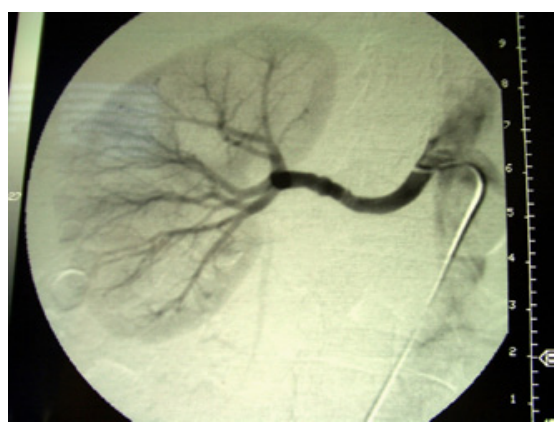

Figure 1: At digital subtraction angiography, the vascular radiologist reported: "mild irregularities (or spasms?) at the middle segment of the renal artery wall" in the right kidney".

In no cases the characteristic signs of FMD, as string-of-beads , segmental stenoses or aneurysms, were described by the vascular radiologist. The angiography data defined the presence of "mild irregularities (or spasms) at the middle segment of the renal artery wall" only in 3 cases, two of them seen at standard angiography Figure 1 , the other at CTA.

In two other cases, the favorable opinion of the vascular radiologist on the perfect viability of the vascular supply was based on the attribution of the mild irregularities of the renal artery to spasms secondary to an over quick introduction of the contrast medium into the artery. The transplant surgeon, after examining highly magnified images of these cases noticed that the very slight irregularities were at the median segment of the artery, in other words in the typical site of FMD and that they remained immobile at every radiological image. She felt it was necessary to obtain a specific informed consent to harvest the potentially diseased kidney. Furthermore, she obtained informed consent regarding possible renovascular hypertension deriving from the onset of FMD in the remnant kidney. 
Citation: Berardinelli L, Beretta C, Giussani A (2012) Living Donor Transplantation of Kidneys with Fibromuscular Dysplasia: Indications, Surgical Techniques and Long Term Results in 11 Cases. J Nephrol Ther S4:008. doi:10.4172/2161-0959.S4-008

Page 3 of 6

In six out of these 11 cases, FMD was an incidental intra-operative finding. Two bilateral renal arteries were present in 3 of these cases, and the kidney side was chosen according to smaller size as well as lower GFR evidenced at dynamic renal scintigraphy. All radiographic images and histological findings confirming the presence of FMD, as well as legal documents regarding the informed consent about the possible presence of a FMD and intra-operative findings are kept in our archives and in the Transplant Database.

Arteriogram and intraoperative findings of kidneys with FMD were graded by us using a system which, according to the literature, is commonly adopted and has been published by Indudhara in 1999.2 "Mild" FMD disease was defined as irregularity of the arterial wall without significant stenosis, "moderate" FMD as arterial irregularity with less than $50 \%$ stenosis and "severe" FMD as arterial irregularity

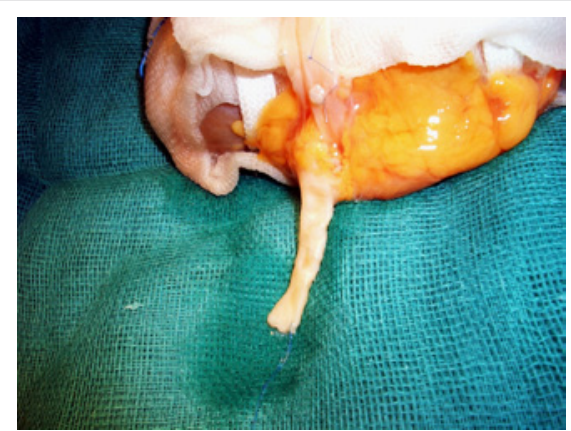

Figure 2: Intra-operative findings of the kidney, whose angiogram was depicted in Figure 1. Initial signs of characteristic "string-of-beads" appearance may be noted in the middle segment of the renal artery.

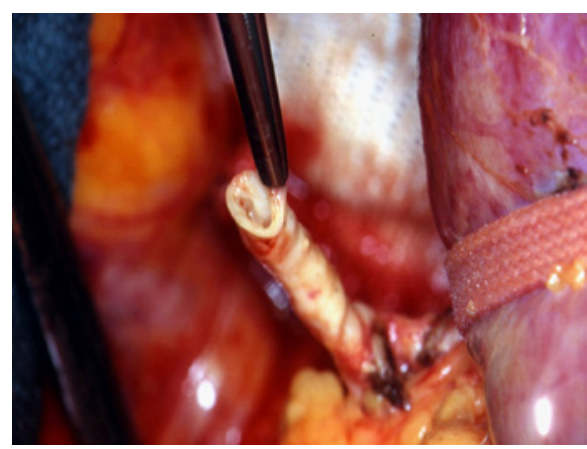

Figure 3: Macroscopical presence of membranes and mild stenoses are evident inside the arterial lumen.

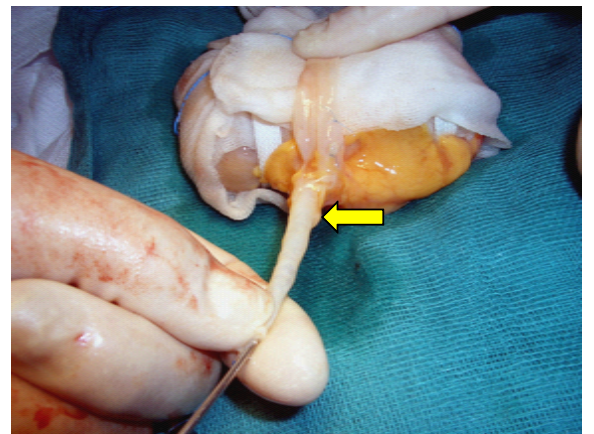

Figure 4: Filling the renal artery with cold perfusional liquid, typical appearance of renal aneurysms may be observed in the middle segment.

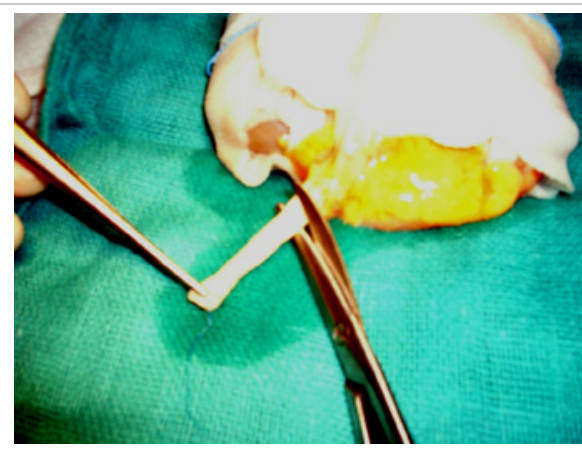

Figure 5: The diseased segment of the renal artery is resected until a healthy enough wall is reached.

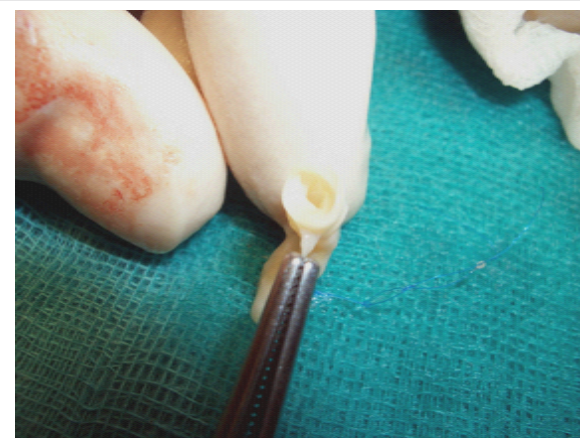

Figure 6: The resected tract of the diseased renal artery shows at the inside multiple delicate membranes, causing mild stenoses.

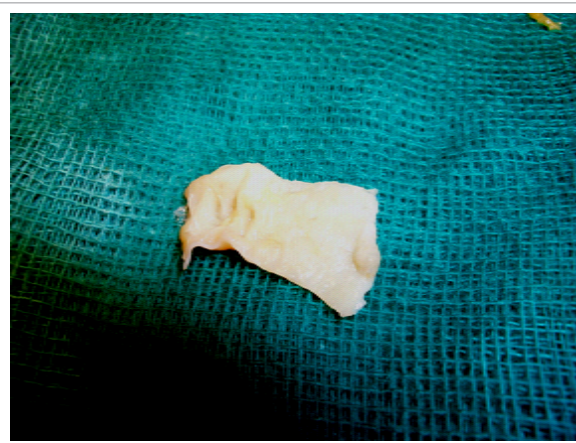

Figure 7: Initial typical signs of FMD can be observed into the renal artery opened.

with more than $50 \%$ stenosis. Other intra-operative findings that had led us to the diagnosis of FMD were segmental rigidity of the artery wall and macroscopical presence of delicate membranes followed by slight stenosis inside the arterial lumen (Figure 2-Figure 7).

With regard to the surgical technique, in 4 grafts with a single renal artery the diseased tract of the renal artery was resected (Figure 5) and anastomosed without any further reconstruction, end-toend to the internal iliac artery (3 cases) or end-to-side to external iliac artery (1 case) of the recipient. Other four grafts with a single renal artery required a special renovascular reconstruction at bench, because of the impossibility to use the recipient hypogastric artery due to atherosclerosis. After having excised the dysplastic lesion, the shortened artery was anastomosed to a biological patch (treated bovine pericardium in 2 cases, homologous saphenous vein in one patient and ovine collagen prosthesis with an intruded Dacron mesh in one 
Citation: Berardinelli L, Beretta C, Giussani A (2012) Living Donor Transplantation of Kidneys with Fibromuscular Dysplasia: Indications, Surgical Techniques and Long Term Results in 11 Cases. J Nephrol Ther S4:008. doi:10.4172/2161-0959.S4-008

patient), which was then anastomosed to the recipient external iliac artery (Figure 8- Figure 11). This technique is preferable with shortened arteries in order to avoid potential angulation after anastomosis due to weight of kidney in its final position. The use of "vascular shield" makes the reconstructions at bench more rapid and safe [9]. (Figure 8) 3 grafts with double renal artery were treated as follows: 2 kidneys evidenced both the main renal artery and the polar renal artery affected by dysplastic lesion : the main renal artery and the smaller polar artery were resected until a healthy distal segment was found. Then, the polar artery was anastomosed to the main renal artery in an end-to-side fashion and the unique shortened arterial trunk was anastomosed in an end-to-end fashion to the internal iliac artery.

In the third kidneys with two renal arteries of similar diameter (Figure 12), both of them found dysplastic at operation, we removed the dysplastic segments and then anastomosed the arteries to a

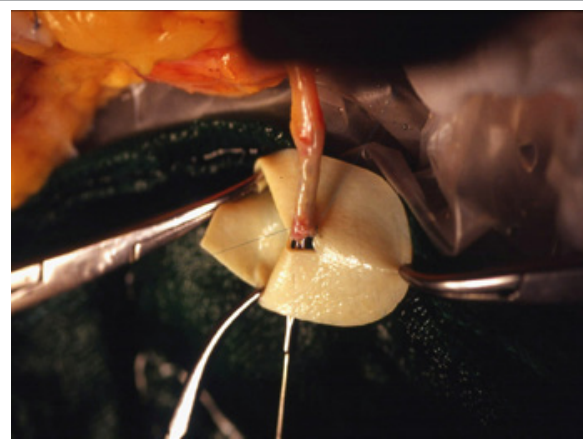

Figure 8: The resected renal artery is now too short for a direct anastomosis and the recipient internal iliac artery is atherosclerotic. Therefore, it is anastomosed end to side to a biological patch. The "vascular shield" (Berardinelli 1978) may be useful to make quicker the reconstruction at bench.

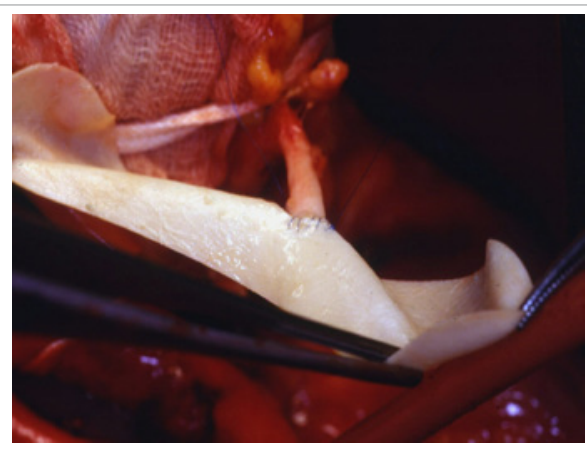

Figure 9:The anastomosis is finished.

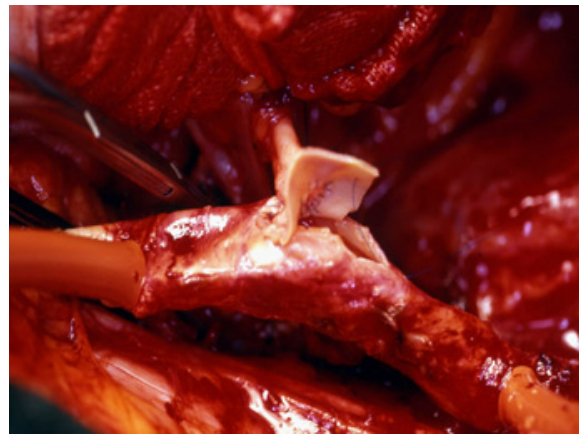

Figure 10: End-to-side anastomosis of the biological patch to the recipient's external iliac artery

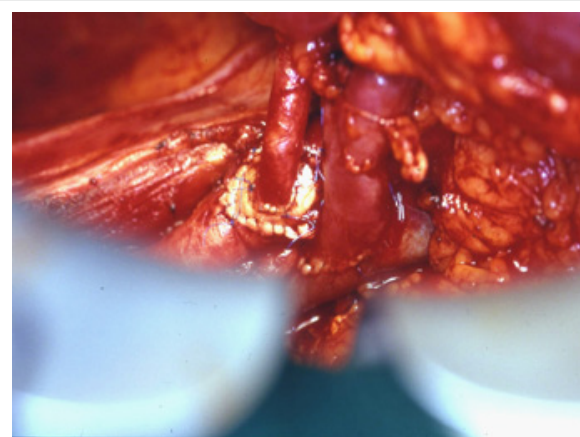

Figure 11: The vascular clamps are removed and the anastomosis of the shortened renal artery to the biological patch can be observed.

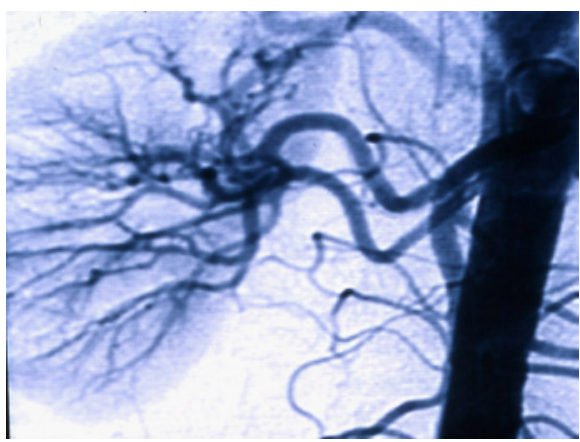

Figure 12: The two renal arteries in the right side appear to be normal at catheter angiogram. At operation, both of them had to be resected due to macroscopical appearance similar to that reported previously in Figure 6 and 7 , and they had became too short for a direct anastomosis. Moreover, the internal iliac artery of the old recipient was not available, due to atherosclerosis.

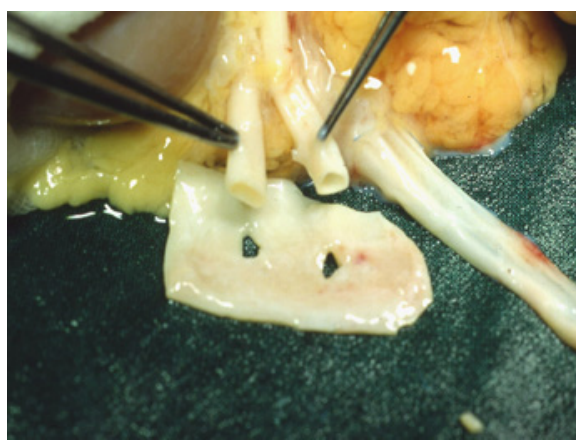

Figure 13: The two renal arteries after resection of the diseased segments are prepared for end to side anastomosis on the biological patch.

biological patch (Figure 13,Figure14) as they had become too short for a direct anastomosis, as aforementioned, and the atherosclerotic internal iliac artery could not be used. Then, in turn, the biological patch was anastomosed to the recipient external iliac artery (Figure 15, Figure 16).

All the arteries reconstructed at bench were tested for a possible hemorrhage, performing an intra-arterial re-perfusion with cold perfusional liquid.

All the 11 grafts showed immediate recovery, without need of dialysis and there were no postoperative complications, in either the donors or in the recipients. One died of lung cancer 7.1 years after transplantation. The mean duration of the grafts was in these recipients 
Citation: Berardinelli L, Beretta C, Giussani A (2012) Living Donor Transplantation of Kidneys with Fibromuscular Dysplasia: Indications, Surgical Techniques and Long Term Results in 11 Cases. J Nephrol Ther S4:008. doi:10.4172/2161-0959.S4-008

Page 5 of 6

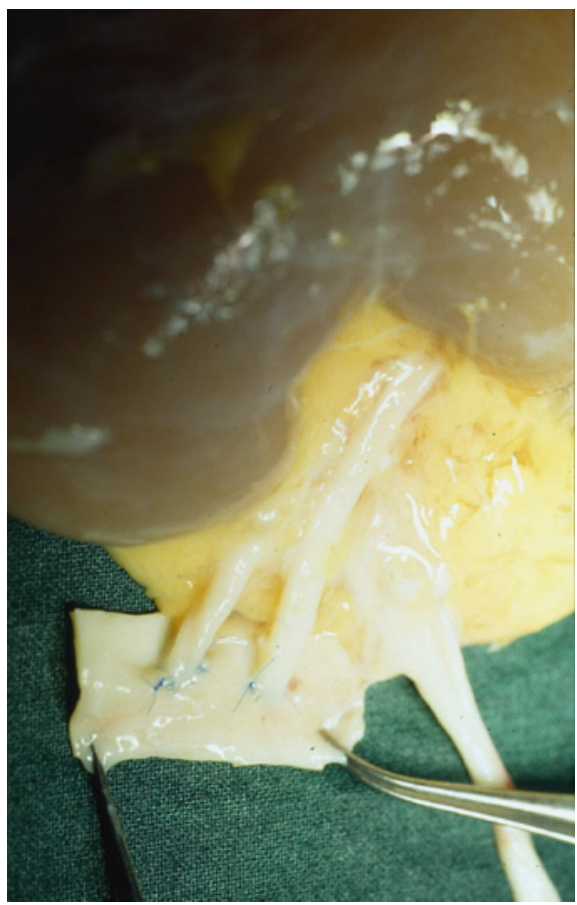

Figure 14: The anastomosis of the two renal arteries on the biological patch is finished.

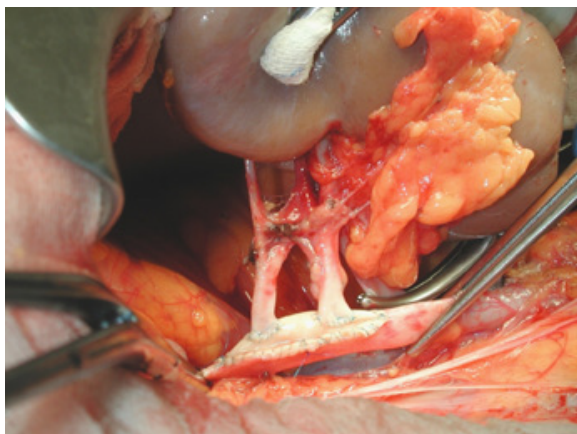

Figure 15: Anastomosis of the biological patch on the external iliac artery.

of $6.3 \pm 4.5$ years for a mean follow-up period of $7.5 \pm 5.9$ yrs (range 1.01-21.6 yrs), very similar to the $6.8 \pm 3.6 \mathrm{yrs}$ of the matched cohort of $117 \mathrm{LD}$-adult recipients (mean follow-up $7.5 \pm 3.9$ yrs, range1.1-21.5 yrs).

Four recipients are well; 3 recipients, who were slightly hypertensive before transplantation (mean systolic blood pressure $142 \mathrm{mmHg}$ and mean diastolic blood pressure $86 \mathrm{mmHg}$ ), continued to show a mild, well controlled hypertension after transplantation (mean systolic blood pressure $144 \mathrm{mmHg}$ and mean diastolic blood pressure 88 $\mathrm{mmHg}$ ). Another recipient became suddenly hypertensive 10 years later due an atherosclerotic stenosis of the hypogastric artery and he was successfully admitted to a PTA. However, no sign of FMD was present in the graft renal artery and the graft continued to function for a further 6 years when it was lost because of chronic allograft nephropathy.

Two other recipients lost their grafts, 1 due to irreversible rejection and 1 from recurrence of the original disease (focal glomerulosclerosis) at 4.9 and 2.4 years, respectively. No recipient with functioning graft has evidenced signs of FMD progression to date.
All donors are well, with normal renal function and all are normotensive, except for the patients who showed mild pre-donation hypertension and remained modestly (mean systolic blood pressure $142 \mathrm{mmHg}$, mean diastolic blood pressure $83 \mathrm{mmHg}$ ) hypertensive (Table 3).

To date none of the donors has had signs of FMD in the remnant kidney. At control we use sophisticated Doppler ultrasound examination as we prefer not to use CTA with potentially nephrotoxic contrast medium. Both donors and recipients remain under close observation for any onset of de-novo renovascular hypertension.

\section{Discussion and Conclusion}

Our study has some limitations, first of all the small number of patients, because of the low incidence of this rare disease in normotensive population. However, the majority of authors (75\%) reporting on donation from LDs affected by FMD [1,3-7,10-15] account for a maximum of 3 cases.

Secondly, the analysis is made using retrospective old data which does not allow radiographic reconstruction or correlation with other radiological techniques and the appearance of FMD could only be verified at the operation.

We have considered 61 cases reported in literature of LD-kidneys affected by FMD and the outcome of the remnant kidney in the donors and of the graft in the recipients $[1,3,4,-7,10-18]$.

Most (81.8\%) of the LDs affected by FMD in our series were women and the mean age was 56 years, which correspond to data reported in literature.

Although the gold standard technique for a preoperative diagnosis of FMD is generally considered to be the intra-arterial angiography with digital subtraction $[1,4,19]$, in our series this procedure was useful to detect this anomaly in only 4 out of 10 donors. Moreover, this test is invasive and does not evidence web-like defects that only become apparent on operation. We now use modern technically sophisticated CTA which evidenced FMD in 50\% of our LDs in this series and has several advantages over traditional angiography, being less invasive and allowing sufficient visualization of vascular supply and organ structures.

A careful evaluation of the whole vascular system is essential for the selection of a suitable organ in LD transplant activity; however, initial renovascular disease may be completely missed at radiographic examinations in asymptomatic donors, as early forms of FMD give rise to no or mild radiographic evidence. Hence the need for detailed

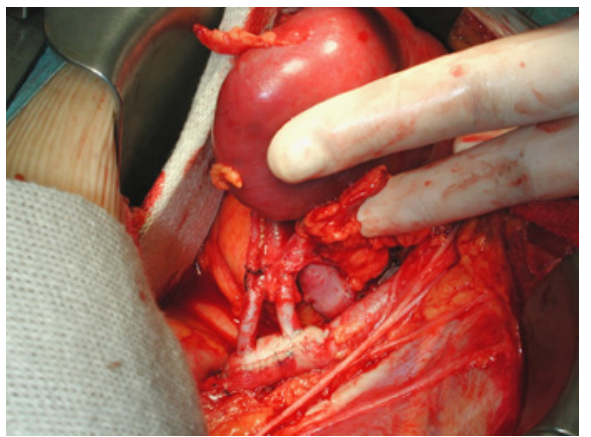

Figure 16: Good appearance of renal parenchyma after removal of vascular clamps. 
Citation: Berardinelli L, Beretta C, Giussani A (2012) Living Donor Transplantation of Kidneys with Fibromuscular Dysplasia: Indications, Surgical Techniques and Long Term Results in 11 Cases. J Nephrol Ther S4:008. doi:10.4172/2161-0959.S4-008

Page 6 of 6

intra-operative observation inside the arterial lumen and the need for reconstruction at bench prior to transplantation using microvascular techniques and the specific armamentarium including magnification devices.

Wider criteria of inclusion have been applied for the LDs in the more recent years of our activity, when we adopted a more aggressive policy of bench reconstructions in kidneys with vascular anomalies: 46 kidneys (25.7\%) out of 179 LD-grafts implanted between January 1996 and November 2011 showed major arterial anomalies, such as multiple renal arteries, heavy renal artery atherosclerosis, fibromuscular hyperplasia or renal aneurysms, most of them (93.7\%) transplanted after a reconstruction at bench.

There are reports in literature, $[10,14,19,20]$ which describe postoperative complications in about $50 \%$ of patients who received grafts with FMD: in these cases, the FMD was not corrected or corrected only by resection of the lesion and a reconstructive microsurgical repair was necessary at the re-operation to save the graft. Better results are reported for grafts treated by an adjunctive interpositional venous graft at bench before transplantation $[3,17,18]$

The techniques we have described showed no complications in the postoperative course, and satisfactory results were observed in the long term, both in the donors and in the recipients.

No signs of FMD were observed in the remnant kidney of our LDs ,as was the case for the patients of Knops [7], Serrano [12], Nahas [13], Wolters [14], Kolettis [16], Balzer [17], and Blondin [18], whilst other authors $[1,4,6]$ each noted signs of hypertension and FMD in the remnant kidney of one LD.

The progression of FMD at various stages and at various postoperative times was observed in only three recipients by Knops [4], Felts [5] and Benavides [6].

In conclusion, candidates with severe or moderate FMD detected in one kidney should not be accepted for donation, because of the tendency to bilateral involvement and possible development of the disease after many years in the remnant or the donated kidney. We completely agree with Parasuraman [1], who speaking about a case of FMD that was undetected by CTA prior to donation, said "Mild FMD in a normotensive potential renal donor cannot be considered a benign condition, as undetected mild stenosis after donation may progress, causing hypertension and compromising the function of the remnant kidney".

However, the use of kidneys from aged living donors with mild, unilateral FMD may be safe for the donor and recipient with careful evaluation, selection and surgical technique. In our experience, reconstruction at bench appears to prevent complications in the recipients. Defining the outcomes implications and most appropriate selection criteria to guide use of kidneys from living donors with mild FMD warrants prospective study with larger samples from diverse centers.

\section{References}

1. Parasuraman R, Attallah N, Venkat KK, Yoshida A, Abouljoud M, et al. (2004) Rapid progression of native renal artery fibromuscular dysplasia following kidney donation. Am J Transplant 4: 1910-1914.

2. Indudhara R, Kenney, Bueschen AJ, Burns JR (1999) Live donor nephrectomy in patients with fibromuscular dysplasia of the renal arteries. J Urol 162: 678681.

3. Borchelt BD, Schweitzer EJ, Bartlett ST (1999) Renal artery reconstruction for Fibromuscolar Dysplasia During a living donor kidney transplant. Vascular Surgery $33: 411-415$

4. Bonatti H, Harthun NL, Sharma R, Brayman K, Sawyer RG, et al. (2010) Fibromuscular dysplasia of the right kidney in a woman who donated her left kidney. Transpl Int 23: e35-38.

5. Felts JH, Whitley NO, Johnston FR (1979) Progression of medial fibroplasia of the renal artery and the development of renovascular hypertension. Nephron 24: $89-90$

6. Benavides CA, Csapo Z, Timmins K, Holley L, Katz SM, et al. (2006) Fibromuscular dysplasia recurrence after kidney transplantation: case report Clin Nephrol 66: 67-70.

7. Knops NB, Cornelissen EA, Monnens LA (2011) Renal transplantation for fibromuscular dysplasia. Am J Transplant 11: 852-856.

8. Lamb EJ, Tomson CR, Roderick PJ; Clinical Sciences Reviews Committee of the Association for Clinical Biochemistry (2005) Estimating kidney function in adults using formulae. Ann Clin Biochem 42: 321-345.

9. Berardinelli L, Vegeto A (1978) A simple vascular shield for microanastomoses Am J Surg 135: 272

10. Linder R, Billing H, Tibell A, Tydén G, Groth CG (1989) Fibromuscular dysplasia in donor kidneys--experience with three cases. Transpl Int 2: 228-231.

11. Cragg AH, Smith TP, Thompson BH, Maroney TP, Stanson AW, et al. (1989) Incidental fibromuscular dysplasia in potential renal donors: long-term clinical follow-up. Radiology 172: 145-147.

12. Serrano DP, Flechner SM, Modlin CS, Streem SB, Goldfarb DA, et al. (1997) The use of kidneys from living donors with renal vascular disease: expanding the donor pool. J Urol 157: 1587-1591.

13. Nahas WC, Lucon AM, Mazzucchi E, Scafuri AG, Neto ED, et al. (1998) Kidney transplantation: the use of living donors with renal artery lesions. J Urol 160: 1244-1247.

14. Wolters HH, Vowinkel T, Schult M, Heidenreich S, Senninger N, et al. (2002) Fibromuscular dysplasia in a living donor: early post-operative allograft artery stenosis with successful venous interposition. Nephrol Dial Transplant 17: 153 155

15. Pfeiffer T, Böhner H, Voiculescu A, Sarbia M, Grabensee B, et al. (2002) Even severe renal artery fibromuscular dysplasia is no contraindication for living donor renal transplantation: report of two successful cases with venous grafting of the donor renal artery. Transplant Proc 34: 3113-3116.

16. Kolettis PN, Bugg CE, Lockhart ME, Bynon SJ, Burns JR (2004) Outcomes for live donor renal transplantation using kidneys with medial fibroplasia. Urology 63: 656-659.

17. Balzer KM, Grotemeyer D, Pfeiffer T, Voiculescu A, Sandmann W (2007) Fibromuscular dysplasia and renal transplantation. Lancet 369: 187.

18. Blondin D, Lanzman R, Schellhammer F, Oels M, Grotemeyer D, et al. (2010) Fibromuscular dysplasia in living renal donors: still a challenge to computed tomographic angiography. Eur J Radiol 75: 67-71.

19. Plouin PF, Perdu J, La Batide-Alanore A, Boutouyrie P, Gimenez-Roqueplo AP, et al. (2007) Fibromuscolar dysplasia. Orphanet J Rare Dis 2: 28

20. Verove C, Bridoux F, Abou-Ayache R, Bauwens M, Drouineau J, et al. (1999) Fibromuscular dysplasia of the allograft renal artery with early post-operative dissection: successful treatment with intravascular stent insertion. Nephrol Dial Transplant 14: 1571-1573. 